with 3 males (60\%). Of the TICI $2 \mathrm{~b} / 3$ patients, there were 7 incidences of vasospasm (27\%), 3 symptomatic intracranial hemorrhages (12\%), 4 hospital deaths (15\%) and 20 favorable outcomes (mRS 0-2 at 90 days) (77\%). In contrast, there were 0 vasospasms, 2 symptomatic intracranial hemorrhages (40\%), 2 hospital deaths (40\%) and only 2 favorable outcomes in the TICI $0 / 2$ a group (40\%). Neither group had any thromboembolic complications. Median (IQR) discharge NIHSS was $2(1-4.75)$ in the TICI $2 \mathrm{~b} / 3$ group compared to 3 (3-40) in the TICI 0/2a group. Both groups shared a median decrease in NIHSS score of 2 post-thrombectomy. Differences between the two groups were not statistically significant.

Conclusion While mild stroke patients with TICI $2 \mathrm{~b} / 3$ reperfusion had better outcomes across the board, the differences were not statistically significant. Mechanical thrombectomy may confer benefits upon mild stroke patients with large vessel occlusion; however, additional research is needed to validate these benefits.

Disclosures F. Chin: None. M. Waqas: None. H. Shallwani: None. H. Rai: None. E. Levy: 1; C; Medtronic US SWIFT PRIME Trials. 2; C; Pulsar Vascular. Advisory Board- Stryker, NeXtGen Biologics, MEDX, Cognition Medical.. 4; C; Intratech Medical Ltd., NeXtGen Biologics.. 6; C; Abbott Vascular for carotid training sessions. A. Siddiqui: 1; C; Cerenovus
LARGE Trial, ARISE II Trial, Medtronic SWIFT PRIME and SWIFT DIRECT Trials, MicroVention FRED Trial \& CONFIDENCE Study, MUSC POSITIVE Trial, Penumbra 3D Separator Trial, COMPASS Trial etc.. 2; C; Amnis Therapeutics, Boston Scientific, Canon Medical Systems USA Inc., Cerebrotech Medical. Systems Inc., Cerenovus, Claret Medical, Corindus Inc., Endostream Medical Ltd etc.. 4; C; Amnis Therapeutics, Apama Medical, BlinkTBI Inc., Buffalo Technology Partners Inc, Cardinal Health, Cerebrotech Medical Systems Inc, Claret Medical, Coggnition Medical, Endostream Medical Ltd etc.

\section{0-006 READMISSION AFTER TREATMENT OF ASYMPTOMATIC CAROTID STENOSIS: A NATIONWIDE READMISSION DATABASE ANALYSIS}

${ }^{1} \mathrm{P}$ Nazari ${ }^{*},{ }^{1} \mathrm{P}$ Golnari, ${ }^{1} \mathrm{R}$ Garcia, ${ }^{1} \mathrm{H}$ Weiss, ${ }^{2} \mathrm{~S}$ Ansari, ${ }^{2} \mathrm{~A}$ Shaibani, ${ }^{2} \mathrm{M}$ Hurley, ${ }^{1} \mathrm{M}$ Potts, ${ }^{1} \mathrm{~B}$ Jahromi. ${ }^{1}$ Neurosurgery, Northwestern University, Chicago, IL; ${ }^{2}$ Radiology, Northwestern University, Chicago, IL

10.1136/neurintsurg-2019-SNIS.6

Introduction Carotid endarterectomy (CEA) and carotid artery stenting (CAS) are two common methods for treating

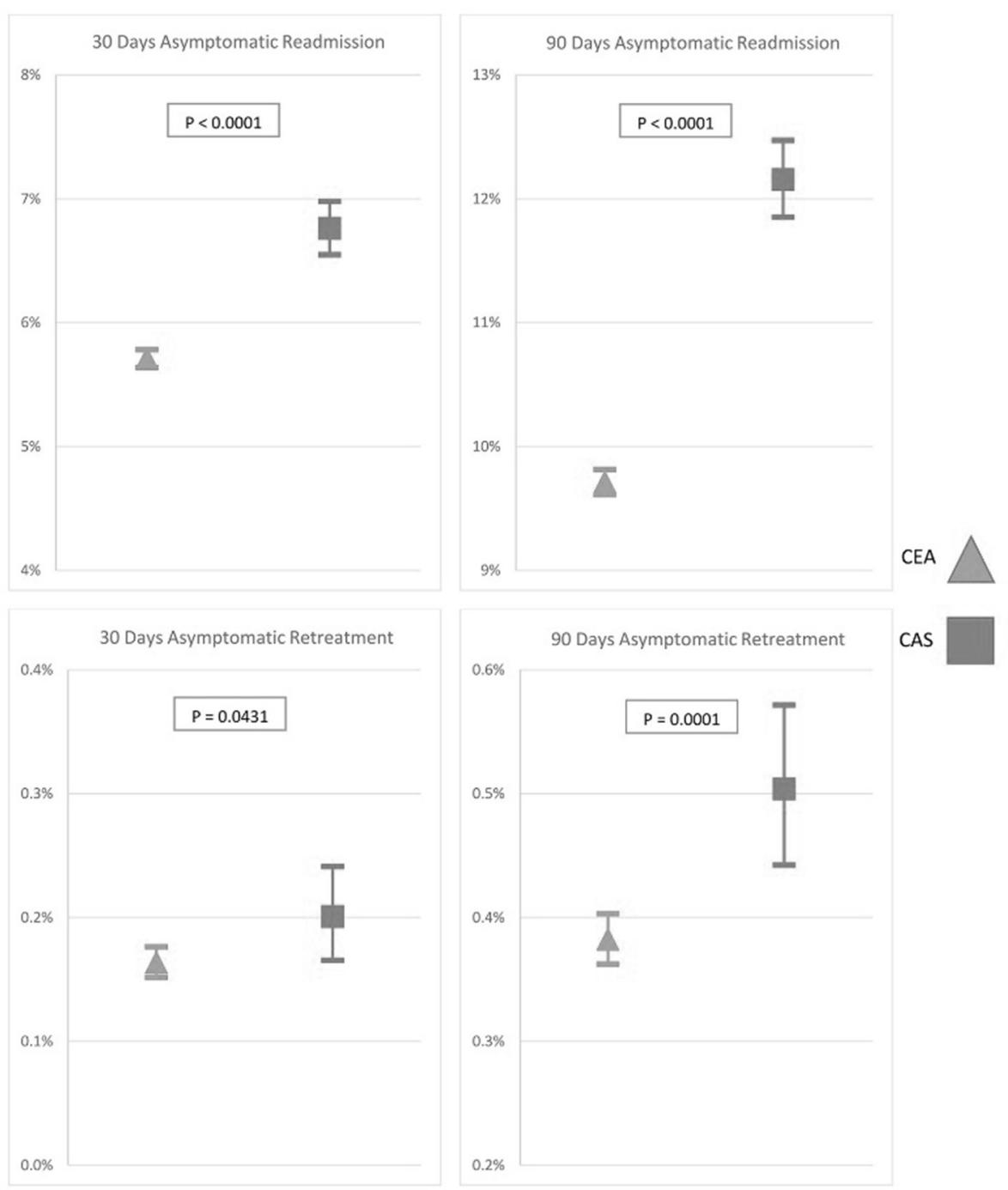


symptomatic and asymptomatic carotid artery stenosis. However, existing literature on readmission after these procedures is limited. In this study, we characterize the rate and causes of 30 and 90-day unplanned readmissions after CEA and CAS for patients with asymptomatic stenosis.

Materials and Methods Data was extracted from the Nationwide Readmission Database spanning 2010 to 2015. The patient population consisted of adult patients who underwent CEA or CAS with a primary diagnosis of occlusion and/or stenosis of a carotid artery without mention of cerebral infarction or TIA. Non-elective readmission within 30 and 90 days were identified and readmission/retreatment rates for CEA vs CAS were also compared.

Results Of 485,838 patients treated and discharged alive, $28,336(5.8 \%)$ patients were readmitted within 30 days, and $41,042(10.0 \%)$ patients were readmitted within 90 days. The most common primary diagnoses for non-elective readmission within 30 and 90 days, respectively, were cerebral artery occlusion with infarct $(3.9 \%, 3.5 \%)$, carotid artery stenosis/ occlusion without infarction $(2.8 \%, 4 \%)$, myocardial infarction $(3.7 \%, 3.5 \%)$, hematoma $(3.5 \%, 2 \%)$, TIA $(2.9 \%, 2.7 \%)$, septicemia $(2.9 \%, 3 \%)$ pneumonia $(2.9 \%, 3 \%)$ and acute kidney failure $(2.1 \%, 2.4 \%)$. The 30 and 90 day non-elective readmission rate for CEA vs CAS was $5.7 \%$ vs $6.8 \%(\mathrm{p}<0.0001)$ and $9.7 \%$ vs $12.2 \%(\mathrm{p}<0.0001)$, respectively. The 30 and $90-$ day non-elective retreatment rates for CEA vs CAS were $0.16 \%$ vs $0.20 \% \quad(p=0.0431)$ and $0.38 \%$ vs $0.50 \%$ $(\mathrm{p}=0.0001)$, respectively (figure 1$)$.

Conclusion Common reasons for 30 and 90-day non-elective readmission after CEA or CAS for asymptomatic stenosis were cerebral artery occlusion with infarct, myocardial infarction and hematoma. Rates of non-elective readmission/retreatment after 30 and 90-days were higher for CAS than CEA.

Disclosures P. Nazari: None. P. Golnari: None. R. Garcia: None. H. Weiss: None. S. Ansari: None. A. Shaibani: None. M. Hurley: None. M. Potts: None. B. Jahromi: None.

\section{0-007 AN ANALYSIS OF STROKE THROMBECTOMY INTERHOSPITAL TRANSPORTATION MODALITY}

H Dasenbrock*, A Beer-Furlan, A Vargas, J Connors, R Crowley, M Chen. Neurosurgery, Rush University, Chicago, IL

\subsection{6/neurintsurg-2019-SNIS.7}

Objective Expeditious interhospital transport of patients with potential large-vessel occlusions is key in the hub and spoke model, where patients are first taken to a local primary hospital to be evaluated for intravenous thrombolysis, and then subsequently transferred to an endovascular capable stroke center. The decision on transport modality-air versus ground transportation-may be multifactorial, dependent upon dispatch times, availability, and cost. This study aims to evaluate and quantify the presumed reduction in time to thrombectomy with air compared to ground transport.

Methods Patients undergoing mechanical thrombectomy for carotid circulation occlusion within 6 hours at an urban, comprehensive stroke center were retrospectively analyzed. Multivariable linear regression evaluated the relationship between transport modality and the time from last known well to groin puncture after adjusting for distance from the comprehensive stroke center.
Results From January 2015 to March 2018, 133 mechanical thrombectomy interhospital transfers were identified; transportation modality was air in $30.8 \% \quad(n=41)$ and ground in $69.2 \%(\mathrm{n}=92)$. The mean inter-hospital distance was 24.1 (standard deviation 16.4, range 0-62) miles. Among patients travelling greater than 10 miles, the use of air transport was associated with a significantly shorter time between last known well and groin puncture when compared to ground (by 26.3 minutes, 95\% CI: 1.1-51.9 minutes, $p=0.04)$. The benefit of air transport was greater with increasing distances, with a significantly shorter time to thrombectomy of 35.1 minutes $(p=0.02)$ if an inter-hospital distance of greater than 20 miles, and of 42.2 minutes $(p=0.03)$ if greater than 30 miles. Within 10 miles however, all patients were transported by ground.

Conclusions In this single-center analysis, helicopter emergency medical service lead to a shorter time to thrombectomy compared with ground transport. Given the known benefit to earlier revascularization on stroke outcomes, these data support the use of emergency aeromedical services when logistically feasible for stroke thrombectomy interhospital transfers greater than 10 miles.

Disclosures H. Dasenbrock: None. A. Beer-Furlan: None. A. Vargas: None. J. Connors: None. R. Crowley: None. M. Chen: 2; C; Genentech, Pneumbra, Stryker, Medtronic.

\section{0-008 HIGH-FREQUENCY OPTICAL COHERENCE TOMOGRAPHY FOR IMAGING NEUROVASCULAR IMPLANTS IN TORTUOSITY}

A Puri, M Marosfoi, G Ughi, R King, E Langan, J Chueh, M Gounis. Radiology, University of Massachusetts, Worcester, MA

\subsection{6/neurintsurg-2019-SNIS.8}

Introduction Intravascular optical coherence tomography (OCT) has an established role in the diagnosis and image guided treatment of coronary artery disease. However, due to the profile and design of the catheter, these devices are not suitable for routine application in neurointerventional surgery. We aim to demonstrate that new generation high frequency OCT (HF-OCT) can image stents and flow diverters used to treat intracranial aneurysms with acceptable workflow deployed in neurointerventional surgery.

Materials and methods An HF-OCT prototype was built (Gentuity, Sudbury MA) that is compatible with standard microcatheters (0.017'). An in vitro flow loop consisting of a patient-specific vascular replica and using porcine blood as a working fluid was deployed to test contrast infusion protocols necessary to remove blood from the target vascular section. Subsequently, eight pigs were implanted bilaterally with flow diverters and stents. The presence of acute platelet aggregation on the surface of the devices and malapposition of the device to the vessel wall was assessed by 3 raters reviewing digital subtraction angiography (DSA), high resolution cone beam CT (CBCT), and HF-OCT. Finally, using the tortuous porcine brachial artery, HF-OCT was performed, and image quality assessed.

Results In the in vitro experiment, the optimized contrast infusion protocol was delivery of contrast through the intermediate catheter (0.058' Navien, Medtronic Neurovascular, Irvine CA) delivering the HF-OCT device at a rate of $5 \mathrm{ml} / \mathrm{s}$ for 20-25 ml of contrast. This protocol resulted in complete 